\title{
A Generalized Architecture for the Frequency- Selective Digital Predistortion Linearization Technique
}

\author{
Jiwoo Kim, Student Member, IEEE, Patrick Roblin, Member, IEEE, Dominique Chaillot, and
} Zhijian Xie, Member, IEEE

\begin{abstract}
This paper presents a new architecture for the frequency-selective digital predistortion (DPD) for two- and three-band power amplifier (PA) linearization. Also, largely spaced-signal DPD using a digital intermediate frequency (IF) technique is demonstrated. The algorithm used accounts for differential memory effects up to fifth order for bands that can be arbitrarily spaced. The simulation and experimental studies are performed using various signal sets; two- and three-band multitone signals with various tone spacing, band separation, and complementary cumulative distribution function. An improvement of $10 \mathrm{~dB}$ over third-order linearization is demonstrated in simulation for more than $20 \mathrm{~dB}$ of adjacent channel power ratio reduction. The test signal and the linearization algorithm were implemented on a field-programmable gate array. The linearization algorithm was applied to an RF amplifier at 700-900 MHz. For the two-band case, more than $15 \mathrm{~dB}$ on the in-band, $13 \mathrm{~dB}$ on the third, and $5 \mathrm{~dB}$ on the fifth intermodulation distortion (IMD) cancellation were achieved. For the three-band case, more than 12 dB of IMD cancellation was observed. For largely spaced signal DPD, more than $15 \mathrm{~dB}$ of IMD cancellation was achieved. In the three-band case, the linearization of intermodulation byproducts overlapping with the in-band distortion is found to be of critical importance.
\end{abstract}

Index Terms-Digital predistortion (DPD), memory effect, multiband linearization, power amplifier (PA).

\section{INTRODUCTION}

$\mathbf{T}$ HE linearization of RF transmitters is one of the most important and challenging issues in modern wireless communication systems since higher linearity is necessary to deliver signals with low error probability. Linearization is also a critical requirement for increasing the capacity of base-stations, thus enabling more economic cell planning.

Manuscript received October 02, 2012; revised October 23, 2012; accepted October 24, 2012. Date of publication December 12, 2012; date of current version January 17, 2013. This work was supported in part by the National Science Foundation under Grant ECS 1129013. This paper is an expanded paper from the IEEE MTT-S International Microwave Symposium, Montreal, QC, Canada, June 17-22, 2012.

J. Kim and P. Roblin are with the Department of Electrical and Computer Engineering, The Ohio State University, Columbus, OH 43210, USA (e-mail: kimji@ece.osu.edu; roblin@ece.osu.edu).

D. Chaillot is with the CEA, 91191 Gif sur Yvette, France (e-mail: d.chaill@wanadoo.fr).

Z. Xie is with the Department of Electrical Engineering, North Carolina A\&T University, Greensboro, NC 27411 USA (e-mail: zxie@ncat.edu).

Color versions of one or more of the figures in this paper are available online at http://ieeexplore.ieee.org.

Digital Object Identifier 10.1109/TMTT.2012.2229714
Memory polynomials have been successfully introduced [1] to compensate for memory effects, and several subsequent works including [2] adopted them to develop powerful linearization algorithms. However, it is very challenging to apply them to linearize the amplification of two communication bands separated by more than $80 \mathrm{MHz}$. A frequency-selective digital predistortion linearization technique has been proposed and demonstrated for addressing the large differential memory effects associated with arbitrary band spacing [3]. One major advantage of the frequency-selective linearization approach is that, if an amplifier is to amplify two widely separated band (say, for example, $500 \mathrm{MHz}$ ) with 10-MHz bandwidth each, each band can be upconverted by different modulators before being combined and amplified, thus reducing drastically the bandwidth requirement on the predistorter digital-to-analog converters (DACs). However, the frequency-selective predistortion of each band accounts for power amplifier (PA) distortion induced by the combined bands. Furthermore, the channel bandwidth requirement on the receiver for the feedback path of the adaptation is similarly reduced since each band can be linearized independently while accounting for the others. Because the receiver analog-to-digital converter (ADC) bandwidth capabilities are even more constrained than those of the DACs in transmitters, this is another important advantage of the frequency-selective scheme.

In [4], an incremental progress was reported, in which an orthogonal condition was added to the algorithm. With this feature, the in-band and interband linearization could be successfully tuned independently. However, this architecture was limited to third order, did not fully utilize the advantage of the reduced feedback bandwidth requirement, and did not incorporate the time-selective dimension of memory polynomials [5], [8] [9].

In [7], a dual-band linearization using a single PA based on IF subsampling technique was simulated, and 10-dB adjacent channel power ration (ACPR) improvement was achieved.

In [8] and [9] , two identical processing stages were used for two different bands using two different local oscillators (LOs) and combined together before the PA. Those two input bands were assumed to be far enough apart so that the intermodulation terms could be removed easily using a filter. In these works, linearization for a band separation of $100 \mathrm{MHz}$ and $1 \mathrm{GHz}$ was demonstrated using two separate vector signal generators. Only in-band linearization was addressed, and 10-16-dB and 12-dB 
ACPR improvements were obtained, respectively, depending on the various signal scenarios.

In [10], the linearization of a $40-\mathrm{MHz}$ band separation with two carriers per band was performed, and about 20-dB ACPR improvement was achieved. Only the linearization of the in-band terms was considered, and the system was demonstrated using a single vector signal generator.

In this paper, a new architecture for the two-band frequencyselective predistortion linearization technique will be presented that extends the original frequency-selective algorithm to higher orders. A preliminary account of the fifth-order extension was presented in the IMS presentation [6]. Note that this approach can be further modified using memory polynomials to address in-band memory effects in each individual band as was proposed in [5] and demonstrated in [8], [9]. In this paper, we further generalize the proposed algorithm to handle multiple bands. As an example, the predistortion linearization of a threeband system will be studied using both simulations and experimental verification. This work will demonstrate that both fifth-order nonlinearities and interband corrections play a key role in multiband systems. The impact of fifth-order nonlinearities on the intermodulation distortion (IMD) will also be studied theoretically. Finally, a $250-\mathrm{MHz}$ spaced signal linearization scheme using digital complex IF technique in which only a single LO is needed for the band separation will be presented. All of the test signal generation and the real-time linearization algorithm for the three different DPD experiments targeted will be implemented using a low-cost field-programmable gate array (FPGA).

This paper is organized as follows. In Section II, the principle of the new algorithms is presented and verified using computer simulations. In Section III and IV, the FPGA implementation of the algorithm is described, and experimental results are presented for each of two-band, three-band, and largely spaced band cases, respectively. In the conclusion, the results achieved from the theory and experiments are summarized.

\section{THEORY OF FREQUENCY-SELECTIVE DPD LINEARIZATION}

\section{A. Two-Band Case}

Let us consider two separate frequency bands represented each by a pair of $I$ and $Q$ baseband signals: $I_{L}$ and $Q_{L}=\widehat{I_{L}}$ for the lower band and $I_{U}$ and $Q_{U}=\widehat{Q_{L}}$ for the upper band. The hat notation is used to signify a Hilbert transform. Assuming that the bands are located at frequency $f_{1}$ and $f_{2}$ respectively, the third-order intermodulation introduces spurious bands at $2 f_{1}-f_{2}$ and $2 f_{2}-f_{1}$. The fifth-order intermodulation introduces additional spurious bands at $3 f_{1}-2 f_{2}$ and $3 f_{2}-2 f_{1}$.

In [3] and [4], an orthogonal two-band frequency-selective linearization scheme was introduced and demonstrated. In that algorithm, the linearization of an amplifier with third-order nonlinearities relied on six complex coefficients to compensate for the in-band and interband (IMD) distortion independently.

Let us first consider the generation of the fundamental thirdand fifth-order IMD components. Given the $\left(I_{L}, Q_{L}\right)$ and $\left(I_{U}, Q_{U}\right)$ lower and upper baseband signals, we shall call $\left(I_{-3}, Q_{-3}\right)$ and $\left(I_{3}, Q_{3}\right)$ the associated lower and upper baseband signals introduced by the third-order intermodulation at the spurious bands $2 f_{1}-f_{2}$ and $2 f_{2}-f_{1}$, respectively. Similarly, we shall call $\left(I_{-5}, Q_{-5}\right)$ and $\left(I_{5}, Q_{5}\right)$ the associated lower and upper baseband signals introduced by the fifth-order intermodulation at the spurious bands $3 f_{1}-2 f_{2}$ and $3 f_{2}-2 f_{1}$, respectively.

For the third- and fifth-order cases, these IMD terms are readily found from Volterra theory [5] to be given by

$$
\begin{aligned}
I_{-5}= & I_{L}^{3} I_{U}^{2}-I_{L}^{3} Q_{U}^{2}+6 I_{L}^{2} Q_{L} I_{U} Q_{U}-3 I_{L} Q_{L}^{2} I_{U}^{2} \\
& +3 I_{L} Q_{L}^{2} Q_{U}^{2}-2 Q_{L}^{3} I_{U} Q_{U} \\
Q_{-5}= & Q_{L}^{3} Q_{U}^{2}-Q_{L}^{3} I_{U}^{2}+6 Q_{L}^{2} I_{L} Q_{U} I_{U}-3 Q_{L} I_{L}^{2} Q_{U}^{2} \\
& +3 Q_{L} I_{L}^{2} I_{U}^{2}-2 I_{L}^{3} Q_{U} I_{U} \\
I_{-3}= & \left(I_{L}^{2}-Q_{L}^{2}\right) I_{U}+2 I_{L} Q_{U} Q_{L}, \\
Q_{-3}= & -\left(I_{L}^{2}-Q_{L}^{2}\right) Q_{U}+2 I_{U} I_{L} Q_{L}, \\
I_{3}= & \left(I_{U}^{2}-Q_{U}^{2}\right) I_{L}+2 I_{U} Q_{L} Q_{U}, \\
Q_{3}= & -\left(I_{U}^{2}-Q_{U}^{2}\right) Q_{L}+2 I_{U} I_{L} Q_{U}, \\
I_{5}= & I_{U}^{3} I_{L}^{2}-I_{U}^{3} Q_{L}^{2}+6 I_{U}^{2} Q_{U} I_{L} Q_{L}-3 I_{U} Q_{U}^{2} I_{L}^{2} \\
& +3 I_{U} Q_{U}^{2} Q_{L}^{2}-2 Q_{U}^{3} I_{L} Q_{L}, \\
Q_{5}= & Q_{U}^{3} Q_{L}^{2}-Q_{U}^{3} I_{L}^{2}+6 Q_{U}^{2} I_{U} Q_{L} I_{L}-3 Q_{U} I_{U}^{2} Q_{L}^{2} \\
& +3 Q_{U} I_{U}^{2} I_{L}^{2}-2 I_{U}^{3} Q_{L} I_{L},
\end{aligned}
$$

in addition to $I_{-1}=I_{L}, Q_{-1}=Q_{L}, I_{1}=I_{U}$, and $Q_{1}=$ $Q_{U}$. Note that the equations reported above recast the thirdorder frequency-selective theory in a more compact way than previously reported in [3] while adding the targeted new fifthorder corrections. Appendix I shows the demonstration of this equivalence.

To take full advantage of the differential memory capability of the frequency-selective linearization architecture, the different in-band and interband components $\left(I_{p}, Q_{p}\right)$ must be rescaled and phase-shifted independently using an IQ modulator. The input and output relationships of the IQ modulator for a $p$ th-order component is of the form

$$
\left[\begin{array}{c}
I_{p}^{\prime} \\
Q_{p}^{\prime}
\end{array}\right]=\left[\begin{array}{cc}
\alpha_{p}\left(E_{U}^{2}, E_{L}^{2}\right) & -\beta_{p}\left(E_{U}^{2}, E_{L}^{2}\right) \\
\beta_{p}\left(E_{U}^{2}, E_{L}^{2}\right) & \alpha_{p}\left(E_{U}^{2}, E_{L}^{2}\right)
\end{array}\right]\left[\begin{array}{c}
I_{p} \\
Q_{p}
\end{array}\right]
$$

where $I_{p}, Q_{p}, I_{p}^{\prime}$, and $Q_{p}^{\prime}$ are the inputs and outputs of the IQ modulator, respectively, and $\alpha_{p}$ and $\beta_{p}$ are the complex coefficients used by the modulator. $E_{L}^{2}$ and $E_{U}^{2}$ are the envelope of lower and upper side-band signals, which are given by $E_{L}^{2}=I_{-1}^{2}+Q_{-1}^{2}$ and $E_{U}^{2}=I_{1}^{2}+Q_{1}^{2}$, respectively. The same matrix equation holds also for the in-band components $p= \pm 1$ beside the interband components $|p|>1$. Note that additional envelopes $E_{p}^{2}=I_{p}^{2}+Q_{p}^{2}$ may be introduced for intermodulation linearization as the intermodulation corrections injected in the PA effectively introduces new bands beside the original input bands.

The functional dependence of the $\alpha_{p}$ and $\beta_{p}$ on the envelopes can be expressed using a Taylor expansion. For the case of a two-envelope dependence $E_{U}^{2}$ and $E_{L}^{2}$ and a fifth-order expansion, this gives

$$
\begin{aligned}
\alpha_{p} \simeq & \alpha_{p, 0,0}+\alpha_{p, 0,1} E_{U}^{2}+\alpha_{p, 1,0} E_{L}^{2} \\
& +\alpha_{p, 0,2} E_{U}^{4}+\alpha_{p, 2,0} E_{L}^{4}+\alpha_{p, 1,1} E_{U}^{2} E_{L}^{2} \\
\beta_{p} \simeq & \beta_{p, 0,0}+\beta_{p, 0,1} E_{U}^{2}+\beta_{p, 1,0} E_{L}^{2} \\
& +\beta_{p, 0,2} E_{U}^{4}+\beta_{p, 2,0} E_{L}^{4}+\beta_{p, 1,1} E_{U}^{2} E_{L}^{2} .
\end{aligned}
$$




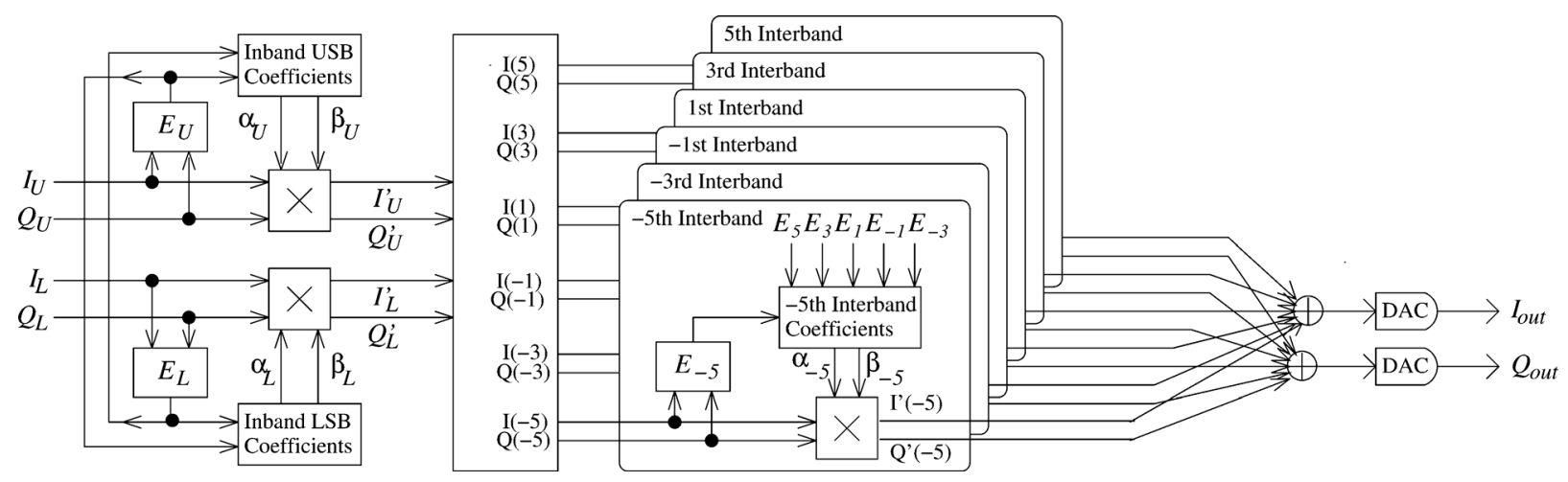

Fig. 1. Proposed frequency-selective DPD architecture.

The final output $I_{\text {out }}$ and $Q_{\text {out }}$ are then given by

$$
I_{\text {out }}(t)=\sum_{\substack{p=-P \\ p \text { is odd }}}^{P} I_{p}^{\prime}(t) \quad \text { and } \quad Q_{\text {out }}(t)=\sum_{\substack{p=-P \\ p \text { is odd }}}^{P} Q_{p}^{\prime}(t) .
$$

The resulting proposed algorithm structure is shown in Fig. 1. Note that it is also beneficial while pursuing intermodulation linearization to perform the in-band linearization first before performing the interband linearization [3]. Subsequent in-band linearization blocks could be added after each IMD component to account for the envelopes associated with the new added IMD bands.

In Section II-C, the importance of a fifth-order term will be analyzed for the case of in the context of the linearization of the three-band spectrum to be discussed in the next section. Further discussion on the impact of the fifth-order nonlinearity on the third-order intermodulation is given in Appendix II.

\section{B. Three-Band Case}

The two-band linearization theory can be extended easily to a larger number of bands where interband linearization plays an important role. Here, a three-band case is presented. Fig. 2 shows the three-band situation with its associated coefficients. For each band, we have

$$
\begin{aligned}
x_{k}(t) & =I_{k}(t)+j Q_{k}(t),(k=L, M, U) \\
x_{k}^{\prime}(t) & =I_{k}^{\prime}(t)+j Q_{k}^{\prime}(t)=\gamma_{k} x_{k}(t) \\
E_{k}(t) & =\left|x_{k}(t)\right| \\
\gamma_{k} & =\alpha_{k}+j \beta_{k}=\gamma_{k}\left(E_{L}^{2}, E_{M}^{2}, E_{U}^{2}\right) \\
& \simeq c_{k, 0}+c_{k, L} E_{L}^{2}+c_{k, M} E_{M}^{2}+c_{k, U} E_{U}^{2}
\end{aligned}
$$

where $k$ is the band index, $x_{k}$ is the complex output signal for the band $k, x_{k}^{\prime}$ is the complex output signal for the band $k$ after its IQ modulator, $E_{k}$ is the envelope of the complex signal $x_{k}$, and $\gamma_{k}$ are the complex analytic functions to be estimated. For simplicity of presentation, a third-order Taylor expansion is presented for $\gamma_{k}$.

The important thing in the three-band case is the interaction of the lowest sideband (LSB) and middle sibeband (MSB), which generates additional interband modulation terms. One of these terms is located exactly in the position of the upper sideband

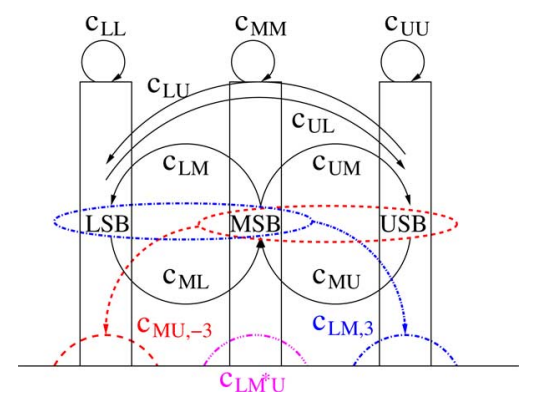

Fig. 2. Three bands with associated linearization coefficients.

(USB) if all three bands are equally spaced. Similarly, the interaction of the MSB and the USB generates an interband term that falls into the location of the LSB. Even if they are not equally spaced, those interband modulation terms are in the band of interest and need to be removed. Furthermore, if each band is getting closer, the band rejection requirement of the RF filter is getting sharper and the cost would be increased steeply. Therefore, interband linearization is an essential block for a multiband linearization.

For the interband linearization, the same formulas could be used as in the two-band case. For example, the interaction of MSB and USB via $x_{M}^{2} x_{U}^{*}$ generates the -third-order IMD term in the location of the LSB, and the interaction of LSB and MSB via $x_{M}^{2} x_{L}^{*}$ generates the +third-order IMD term in the position of the USB. In addition, the three-band product $x_{L} x_{M}^{*} x_{U}$ generates the interband term in the location of the MSB. From the above two- and three-band equations, the following third IMD terms are then obtained:

$$
\begin{aligned}
I_{M U,-3} & =\left(I_{M}^{2}-Q_{M}^{2}\right) I_{U}+2 I_{M} Q_{U} Q_{M} \\
Q_{M U,-3} & =-\left(I_{M}^{2}-Q_{M}^{2}\right) Q_{U}+2 I_{U} I_{M} Q_{M} \\
I_{L M, 3} & =\left(I_{M}^{2}-Q_{M}^{2}\right) I_{L}+2 I_{M} Q_{L} Q_{M} \\
Q_{L M, 3} & =-\left(I_{M}^{2}-Q_{M}^{2}\right) Q_{L}+2 I_{M} I_{L} Q_{M} \\
I_{L M{ }^{*}} & =I_{L} I_{M} I_{U}+I_{L} Q_{M} Q_{U}-Q_{L} I_{M} Q_{U}+Q_{L} Q_{M} I_{U} \\
Q_{L M *} U & =I_{L} I_{M} Q_{U}-I_{L} Q_{M} I_{U}+Q_{L} I_{M} I_{U}+Q_{L} Q_{M} Q_{U}
\end{aligned}
$$

Associated with the above third-order IMD terms are the complex linearization coefficients $c_{M U,-3}, c_{L M, 3}$, and $c_{L M^{*} U}$. Note that two other three-band products $x_{L}^{*} x_{M} x_{U}$ and $x_{L} x_{M} x_{U}^{*}$ can 


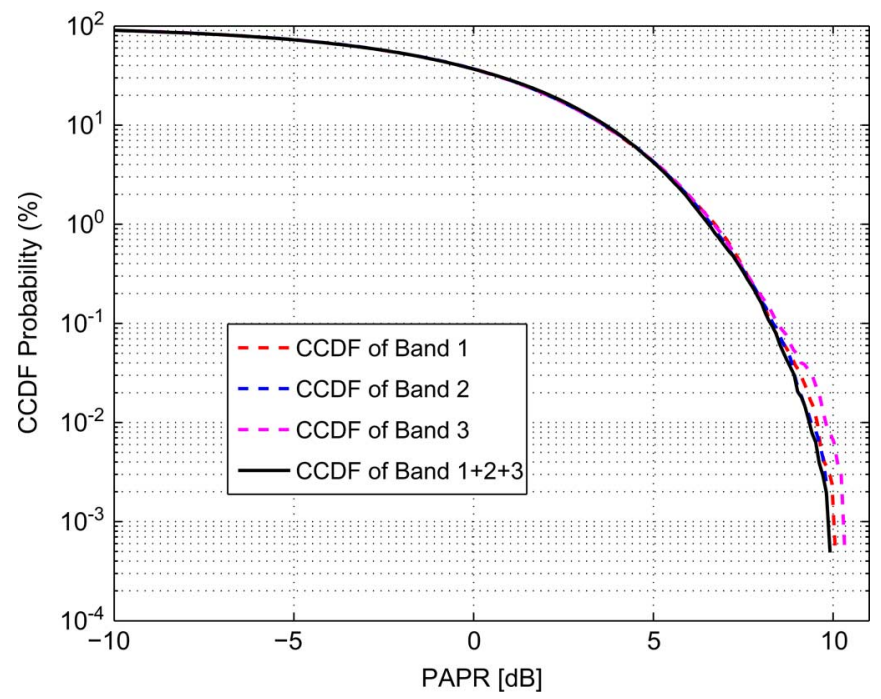

Fig. 3. CCDF of the three uncorrelated LTE bands used as a test.

be identified that also generate interband terms. However, those two IMD terms are all located out of the band of interest (inband is limited to $\mathrm{L}, \mathrm{M}$, and $\mathrm{U}$ ) and can thus be readily removed using conventional filtering for sufficiently large band spacing.

Similarly, the fifth-order IMD terms associated with $x_{L}^{2} x_{M}^{* 2} x_{U}, x_{L}^{*} x_{M}^{3} x_{U}^{*}$, and $x_{L} x_{M}^{* 2} x_{U}^{2}$ can be easily be evaluated for the lower, middle, and upper bands, respectively.

\section{Simulation Verification}

Here, we shall test the efficacy of the proposed multiband fifth-order linearization scheme using simulation. Three longterm evolution (LTE) signals of a 5-MHz bandwidth, centered in noncontiguous bands $20 \mathrm{MHz}$ apart, will be used for the input excitation.

The linearization simulations are conducted in MATLAB using a memoryless PA model with third-order nonlinearities to illustrate the efficacy of the proposed algorithm. The PA transfer characteristics is

$$
y_{\mathrm{OUT}}(t)=x_{\mathrm{IN}}(t)\left(1-\alpha\left|x_{\mathrm{IN}}(t)\right|^{2}\right)
$$

where $x_{\mathrm{IN}}=I(t)+j Q(t)$ and the small-signal gain of the PA is normalized to one.

To best demonstrate the impact of the linearization, the input signal power is adjusted such that the amplifier is driven into a deep compression of $3.5 \mathrm{~dB}$ at the peak input power. Using an input signal $x_{\text {IN }}$ with peak power normalized to one $\left(\left|x_{\mathrm{IN}}\right| \leq\right.$ 1 ), this occurs for $\alpha=G_{n}-1$. Fig. 3 shows the complementary cumulative distribution functions (CCDFs) for the three LTE bands used and for their composite (sum) signal. The peak power is reached when the envelope of the three bands is constructively added. This results in that the peak-to-average power ratio (PAPR) of the composite signal is slightly smaller than the PAPR of the three individual bands. As shown in Fig. 3, the maximum PAPR reaches $10 \mathrm{~dB}$ with a probability of occurrence of $0.001 \%$.

The AM-to-AM characteristic of the PA is shown in Fig. 4. As indicated by the AM-AM curve (Band $1+2+3$ ), the amplifier

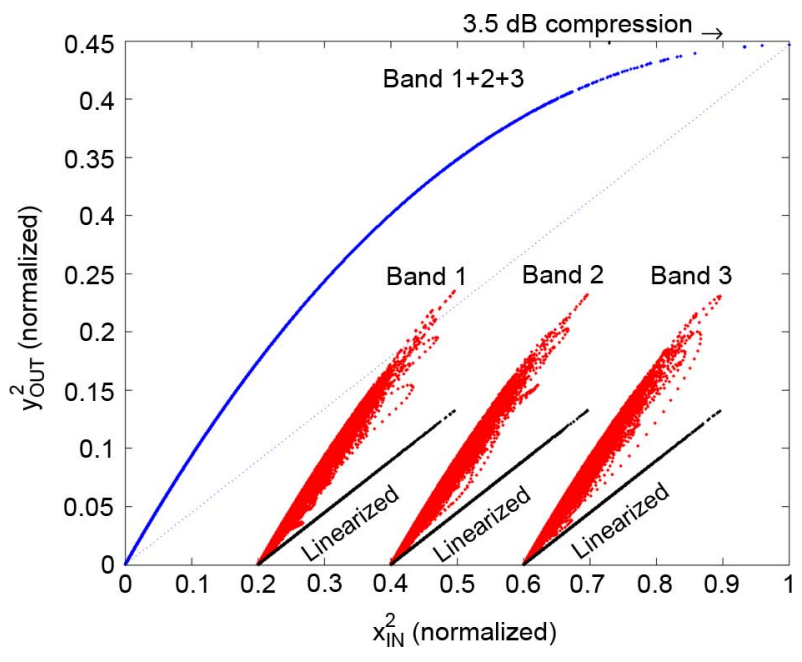

Fig. 4. Simulation results showing the AM-AM characteristics of the test memoryless PA for three LTE signals before (blue and red lines) and after (black lines) predistortion linearization.

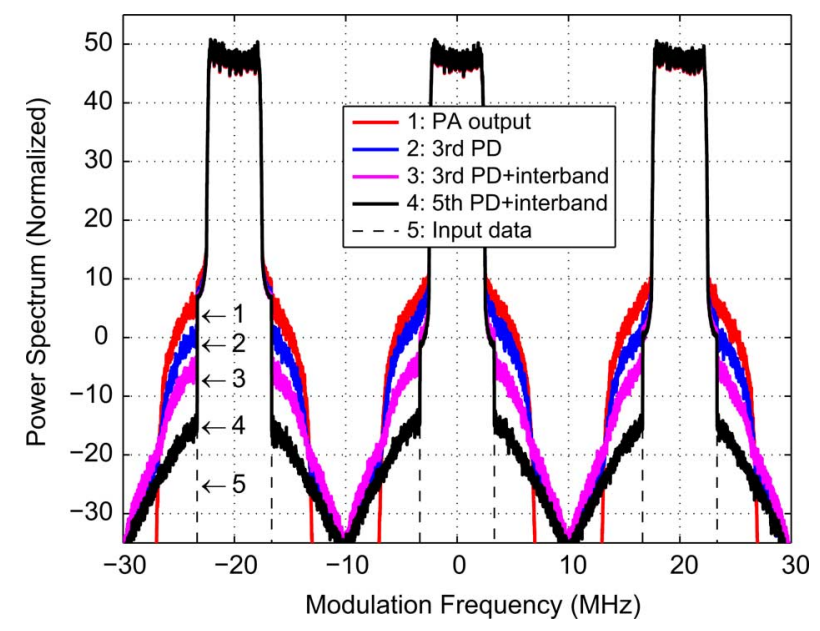

Fig. 5. Simulation results showing the spectra obtained at the PA output without PD (1: red line); with third-order PD without interband (2: blue line); third-order PD with interband (3: magenta line); fifth-order PD with interband (4: black line). The input spectrum (5: black dashed line) is included for reference.

is memoryless but nonlinear. However, the AM-AM characteristics of Bands 1,2, and 3 are individually exhibiting strong memory effects as indicated by their hysteresis. Note that, for the sake of clarity, these AM-AM characteristics were respectively shifted horizontally by $0.2,0.4$, and 0.6 on the normalized input power axis. These results demonstrate that even a memoryless nonlinear PA will exhibit memory effects when excited by a multiple-band signal. Indeed, the PA nonlinearities introduce unwanted intermodulation signals that superpose with the desired inbands signals in the same frequency range. The spectral regrowth associated with the PA is shown in Fig. 5 using the top red curved labeled 1 . For comparison, the spectrum of the input LTE data is shown using a dashed line labeled 5. Note that the LTE signal consists of 204780 samples played in a loop with a transition region of 80 data points to remove any spectral leakage. Spectral video averaging is also used to reduce the spectral noise. 
To demonstrate the linearization of the test third-order PA, the proposed fifth-order predistortion (PD) that inverts the PA characteristic in a least-square sense was extracted. This PD extraction is realized by a linear least-square fit of the input PA signal (PD output) versus the output PA signal (PD input). First, the results obtained for a third-order predistortion neglecting the interband terms $x_{M}^{2} x_{U}^{*}$ and $x_{M}^{2} x_{L}^{*}$ is shown in Fig. 5 using a blue line labeled by 2 . At best, a 5-dB ACPR reduction is observed. The result obtained for a 3rd order predistortion including the interband terms $x_{M}^{2} x_{U}^{*}$ and $x_{M}^{2} x_{L}^{*}$ is shown next in Fig. 5 using a magenta line labeled by 3 . An ACPR reduction of about $10 \mathrm{~dB}$ is observed. Finally the result obtained for a 5 th order predistortion including the 5 th order interband terms $x_{L}^{2} x_{M}^{* 2} x_{U}, x_{L}^{*} x_{M}^{3} x_{U}^{*}$ and $x_{L} x_{M}^{* 2} x_{U}^{2}$ is shown in Fig. 5 using a black line labeled by 4 . An ACPR reduction of at least $20 \mathrm{~dB}$ is observed. Referring back to Fig. 4, one also observes that the three black lines labeled "Linearized" demonstrate that the proposed multiband linearization has indeed linearize the signal measured in bands 1,2 , and 3 . Note that the same power backoff of $3.5 \mathrm{~dB}$ was used in all these cases for a fair comparison of the PA response with and without PD. In summary, these simulation results demonstrate the potential efficacy of the multiband linearization scheme presented here. Note that the linearization algorithm is not limited to memoryless PA but also works for piecewise quasi-memoryless PA when using different complex coefficient $\gamma_{k}=\alpha_{k}+j \beta_{k}$ in (5) and (7) for each band $k$.

\section{HARDWARE ImPlementation AND TeSt SetuP}

\section{A. Signal Selection}

For the system excitation, the input signal could be any bandlimited signals. In the experimental section of this work, twoband multisine signals with a constant amplitude and specific phases have been developed as follows:

$$
\begin{aligned}
I_{U}(t)+j Q_{U}= & \left\{A \sum_{k=1}^{N} e^{j\left(k \Delta \omega t+\phi_{U, k}\right)}\right\} \\
& \times e^{j 2 \pi F_{\mathrm{mod}} t} \\
I_{L}(t)+j Q_{L}= & \left\{A \sum_{k=1}^{N} e^{-j\left(k \Delta \omega t+\phi_{L, k}\right)}\right\} \\
& \times e^{-j 2 \pi F_{\mathrm{mod}} t} .
\end{aligned}
$$

Multisine signal can exhibit a higher PAPR when the individual tones superimpose in phase. In this study, each of the phases $\phi_{U, k}$ and $\phi_{L, k}$ has been optimized so that the total signal achieves a realistic PAPR. In this work, three signal sets have been developed, and Fig. 6 shows the CCDF of the three developed signals: 1) 16-tone per two bands (5-MHz bandwidth for each band) with 7.5-dB PAPR; 2) 96-tone per three bands (5-MHz bandwidth for each band) with 8.7-dB PAPR; and 3) 128-tone for two bands (10-MHz bandwidth for each band) for the largely spaced signal linearization with $8.0-\mathrm{dB}$ PAPR. To demonstrate that the three-band linearization coefficients will

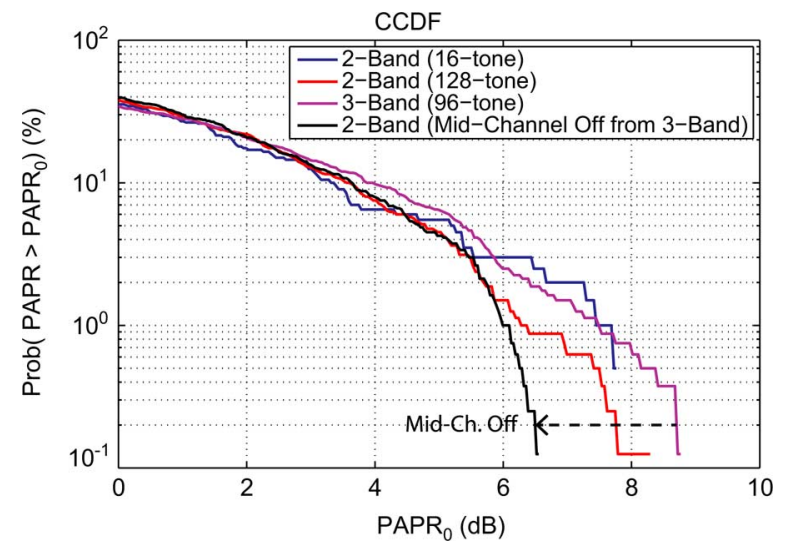

Fig. 6. CCDF plot of 16-, 96- and 128-tone multisine signal.

also work well for two bands, the middle channel will be turned off in Section IV, and the resulting CCDF of the composite signal for the two remaining bands is also plotted in the same figure using a black line.

For the two-band case, a $2 N$-tone composite signal is generated and implemented using lookup tables (LUT) on the FPGA device. For the three-band case, a $3 N$-tone composite signal is generated and implemented on the LUT.

\section{B. Linearization of Up to Fifth-Order IMD}

The overall experimental testbed used in this work is shown in Fig. 7. Multitone signals for each lower and upper sideband signals with specific phases are generated by MATLAB and implemented with an LUT on the Stratix-III 3SE260 FPGA on Altera DE3 board. The FPGA system clock is $125 \mathrm{MHz}$. The predistorted baseband signal from the FPGA is connected to a TI DAC5682z DAC evaluation module (EVM) over low-voltage differential signaling (LVDS). The TI EVM has dual 16-bit DACs with up to 1.0 GSPS, a clock distribution chip, CDCM7005, and analog quadrature modulator, TRF3703, to upconvert the baseband DAC output to RF. Interpolation filter with $\times 4$ rate and the $L C$ low-pass filter (LPF) with $300-\mathrm{MHz} 3-\mathrm{dB}$ corner frequency are used between the DAC and the IQ modulator to remove the Nyquist images from the DAC. dc offset, and IQ-imbalancing have been carefully removed. An RF frequency of $890 \mathrm{MHz}$ was chosen to investigate the digital cellular band response and the external $\mathrm{LO}$ fed into the DAC EVM. The RF output of the EVM is connected via a preamplifier to the Mini-Circuit amplifier ZX60-43-S+ to be linearized.

\section{Linearization of Largely Spaced Signals}

The DAC5682 [11] supports coarse mixing mode that is capable of shifting the input signal spectrum by the fixed mixing frequencies $f_{s} / 2$ or $\pm f_{s} / 4$. For the complex signal $I(t)+j Q(t)$, the output of the coarse mixer block, $I_{\text {out }}(t)$ and $Q_{\text {out }}(t)$ are given by following:

$$
\begin{aligned}
I_{\text {out }}(t) & =I(t) \cos \left(2 \pi f_{\mathrm{IF}} t\right)-Q(t) \sin \left(2 \pi f_{\mathrm{IF}} t\right) \\
Q_{\text {out }}(t) & =I(t) \cos \left(2 \pi f_{\mathrm{IF}} t\right)+Q(t) \sin \left(2 \pi f_{\mathrm{IF}} t\right)
\end{aligned}
$$




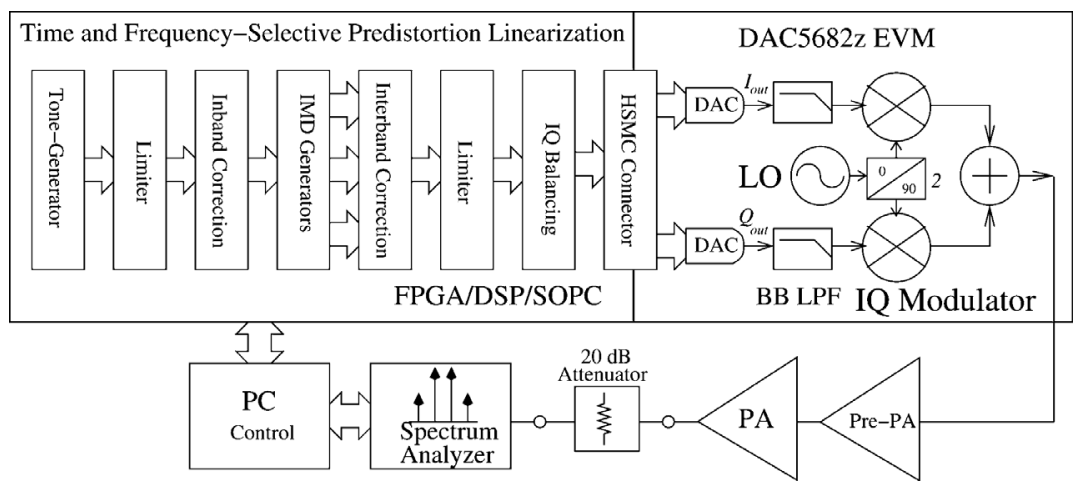

Fig. 7. Testbed setup for the proposed frequency-selective predistortion.

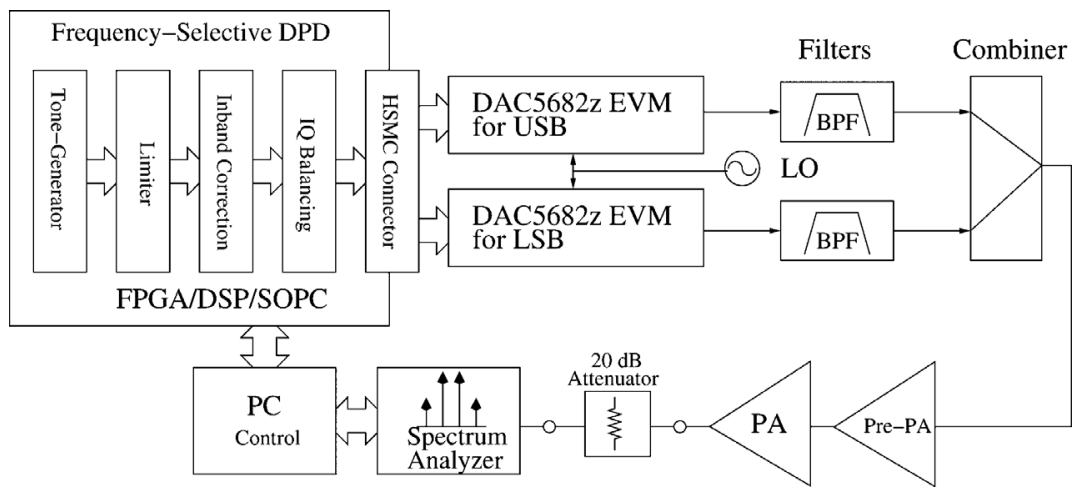

Fig. 8. Largely spaced DPD architecture using digital IF.

where $f_{\mathrm{IF}}$ is the fixed mixing frequency. For example, for the $f_{\mathrm{IF}}=+f_{s} / 4$ case, we have

$$
\begin{aligned}
\left.\cos \left(2 \pi \cdot f_{\mathrm{IF}} \cdot t\right)\right|_{t=n T_{s}} & =\left.\cos \left(2 \pi \cdot \frac{f_{s}}{4} t\right)\right|_{t=n T_{s}} \\
& =\cos \left(\frac{\pi}{2} n\right) \\
& =1,0,-1,0, \ldots \\
\left.\sin \left(2 \pi \cdot f_{\mathrm{IF}} \cdot t\right)\right|_{t=n T_{s}} & =\left.\sin \left(2 \pi \cdot \frac{f_{s}}{4} t\right)\right|_{t=n T_{s}} \\
& =\sin \left(\frac{\pi}{2} n\right) \\
& =0,1,0,-1, \ldots .
\end{aligned}
$$

Therefore, the output sequences for $f_{\mathrm{IF}}=+f_{s} / 4$ case are $I_{\mathrm{out}}=\{+I,-Q,-I,+Q\}$ and $Q_{\mathrm{out}}=\{+Q,+I,-Q,-I\}$. Similarly, for $f_{\mathrm{IF}}=-f_{s} / 4$ case, the output sequences are $I_{\text {out }}=\{+I,+Q,-I,-Q\}$ and $Q_{\text {out }}=\{+Q,-I,+Q,-I\}$.

By connecting additional DAC5682z EVM to the DE3 FPGA board and choosing $f_{\mathrm{IF}}=+f_{s} / 4$ for the USB signal and $f_{\mathrm{IF}}=$ $-f_{s} / 4$ for the LSB signal, the baseband signals are spaced with $2 f_{\mathrm{IF}}$ from each other. In this test, $125-\mathrm{MHz}$ sampling frequency has been used, and, in consequence, a two-band signal with bands separated by $250 \mathrm{MHz}$ has been generated.

The advantages of this architecture is that only a single LO is required for the signal separation and no physical multiplier is needed for the spectrum shifting because the output sequences are just exchanging and/or sign-bit flipping the I and Q signals.

Fig. 8 shows the proposed largely spaced DPD architecture. Since the two bands are largely separated, the interband mod-

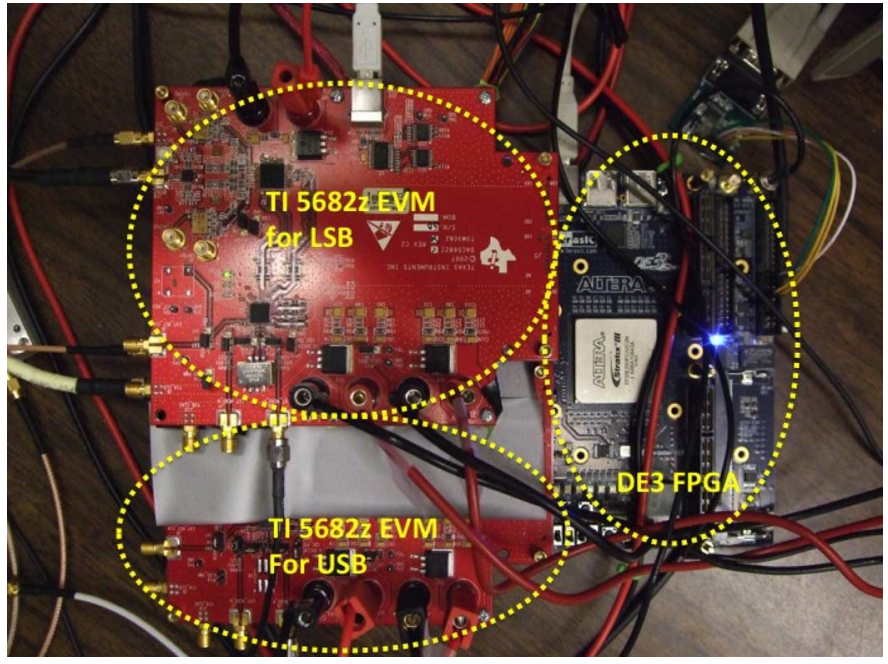

Fig. 9. Photograph of the testbed.

ulation terms are located far enough and are easily removed using filters. Hence, only the in-band linearization part is needed for this test. To remove the images and other unwanted signals, two BPFs have been used. Fig. 9 shows the photograph of the testbed. Only one TI $5682 \mathrm{z}$ EVM is used for the fifth-order linearization and both of the TI EVMs are used for the largely spaced-signal DPD linearization.

\section{EXPERIMENTAL RESULTS}

The following procedure was used for the training: 1) the PA's output power was set so that only the third-order IMD was 


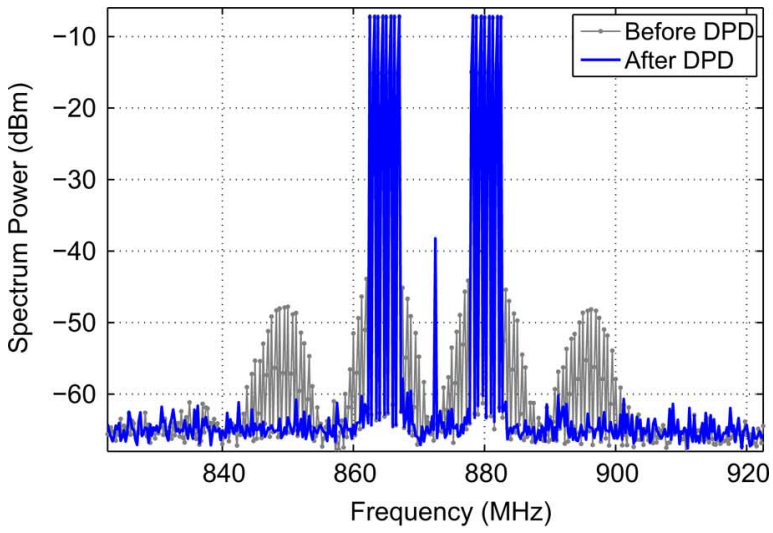

(a)

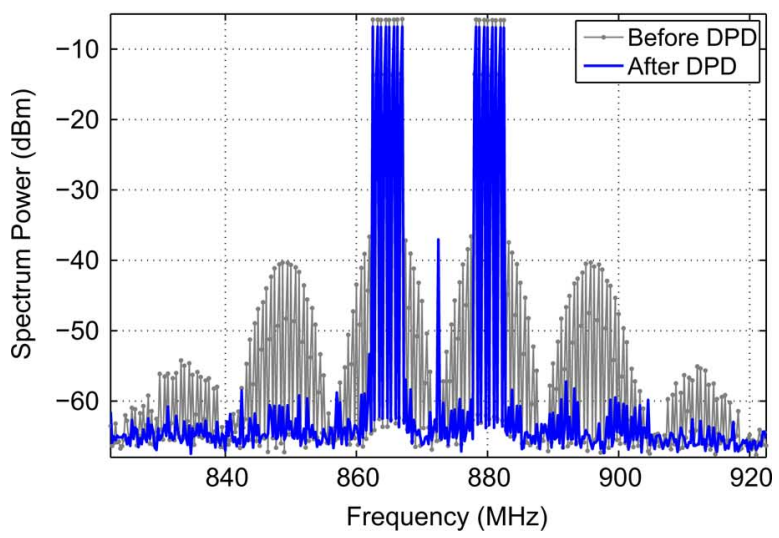

(b)

Fig. 10. Experimental linearization results including both in-band and interband linearization for a 16-tone system per two-band signal at two different power levels (a) up to third order and (b) up to fifth order.

present so that it could be linearized, and 2) the PA's output power was raised so that both the third- and fifth-order IMDs were present to be linearized for both third- and fifth-order IMDs. Taking full advantage of the frequency-selective nature of the algorithm, the linearization was sequentially performed using the following steps: 1) in-band linearization; 2) third-order interband linearization; and 3) fifth-order interband linearization. Fig. 10 shows the result of the two-band linearization of 16-tone over the two-band case up to fifth-order IMD. For the in-band linearization, more than 15-dB IMD cancellation was achieved. For the third-order interband linearization, more than 13-dB IMD cancellation was achieved. Also, for the fifth-order interband linearization, more than 5-dB IMD cancellation is observed in Fig. 10(b). It is to be noted that the LO leakage is not fully removed in Fig. 10. In the subsequent experimental results, we shall make use of a large digital IF so that the LO leakage can easily be removed by filtering beside using the dc offset tuning of the IQ modulator.

For the three-band algorithm, a sequential linearization method similar to the two-band method was used.

Step 1) Turn off MSB and USB while turn on LSB with increased power by a $\sqrt{3}$ ratio to maintain the same output power and then find the self-in-band linearization coefficient, i.e., $c_{L L}$.

Step 2) Repeat step 1) for MSB and USB, i.e., $c_{M M}$ and $c_{U U}$, turning off USB while turning on LSB and MSB with increased power by a $\sqrt{3 / 2}$ ratio so that

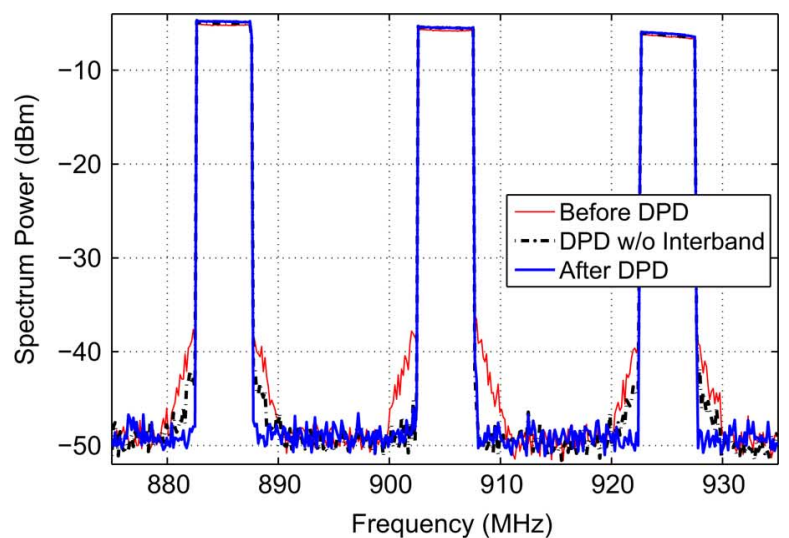

(a)

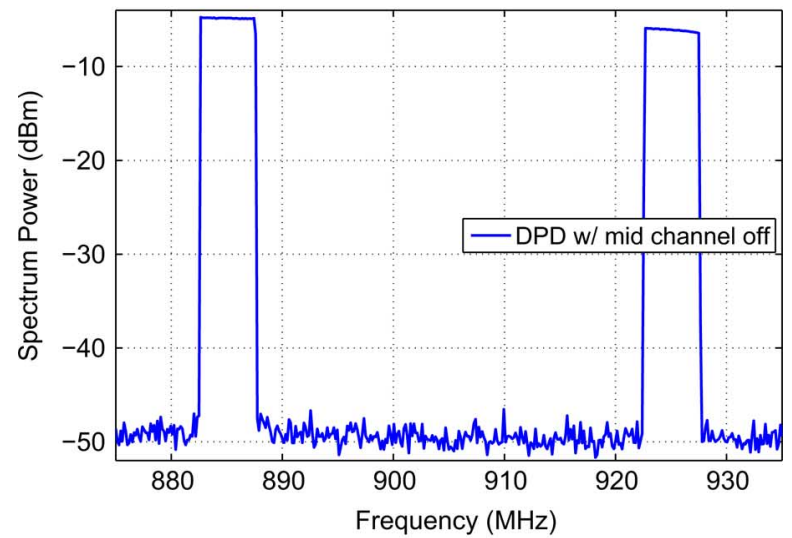

(b)

Fig. 11. (a) Experimental linearization results for a 96-tone per three-band signal with and (a) without interband linearization. (b) Robustness of the linearization is tested by turning off the middle band.

the final output power is the same as for the normal three-band case.

Step 3) Find the two in-band linearization coefficients and the third-order interband linearization coefficient, i.e., $c_{L M}, c_{M L}$, and $c_{L M, 3}$;

Step 4) Repeat step 3) for MSB and USB, i.e., $c_{M U}, c_{U M}$, and $c_{M U,-3}$.

Step 5) Turn on all three bands with normal power and find $c_{L U}, c_{U L}$, and $c_{L M^{*} U}$.

Fig. 11 shows the result of the three-band linearization. Without the interband linearization, it would not be possible to fully linearize the PA output for the three-band signal (black dashed line in the figure). With the interband linearization, more than $12-\mathrm{dB}$ IMD cancellation was achieved.

To investigate the robustness of the DPD system, the midchannel band was turned off. The associated CCDF for the resulting two-band signal is shown in Fig. 6 using black line. Fig. 11(b) shows the spectrum of the mid-channel off from the three-band input. No performance degradation is observed between the two- and three-band cases even though the same linearization coefficients are used. This indicates that the DPD system was robustly identified using the extraction scheme presented above for the three-band signal.

For the largely spaced two-band DPD with digital IF technique, only the in-band linearization was used because the interband terms can be removed easily with an external filter. Fig. 12 


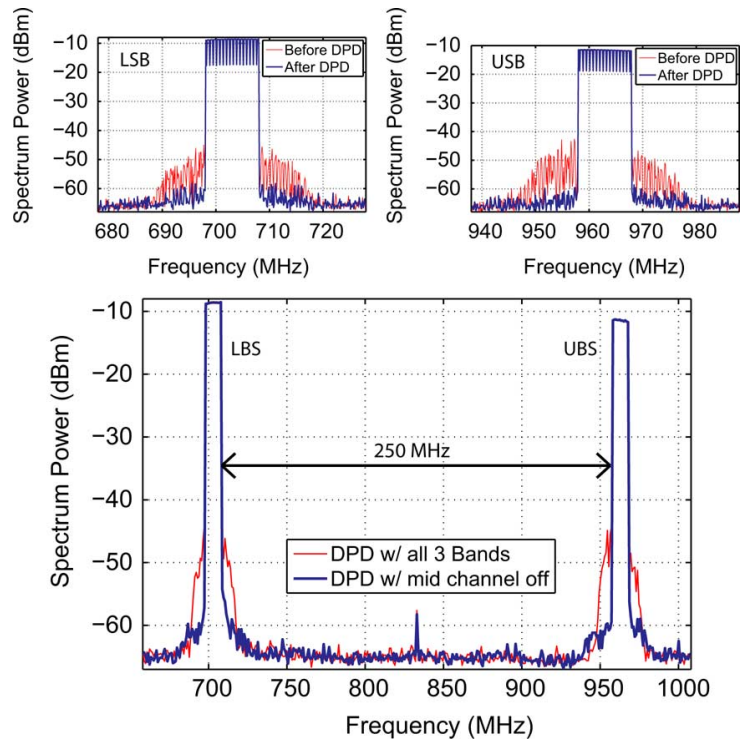

Fig. 12. Experimental linearization results for a 128-tone per two-band signal with $250-\mathrm{MHz}$ band separation. The top plots zoom on the individual linearization.

shows the result of the 250-MHz-spaced signal DPD. For both bands, over 15-dB IMD cancellation was observed. Note that the unequal power levels of LSB and USB are due to the frequency response of the amplifier.

\section{CONCLUSION}

This paper has presented an extended frequency-selective DPD algorithm which could be applied to a larger number of multiple bands. The theory was tested in simulation for a memoryless PA with $3.5 \mathrm{~dB}$ at peak power using three independent 5-MHz LTE signals. A fifth-order linearization was demonstrated in simulation to provide an improvement of 10 $\mathrm{dB}$ over the third-order linearization and more than $20 \mathrm{~dB}$ of overall ACPR reduction.

The theory was also experimentally tested using multisine excitations with realistic CCDFs and PAPR ranging from 7.5 to $8.7 \mathrm{~dB}$. For the two-band case, more than $15 \mathrm{~dB}$ on the in-band, $13 \mathrm{~dB}$ on the third-order interband, and $5 \mathrm{~dB}$ on the fifth-order interband IMD cancellations were achieved. For the three-band case, more than 12-dB IMD cancellations were observed. In the three-band case, the interband linearization was demonstrated as playing an important role. A largely spaced signal two-band DPD using digital IF technique was proposed and more than 15-dB IMD cancellation was achieved.

\section{APPENDIX I}

\section{DEMONSTRATION OF EQUIVALENCE}

In [3], only third-order IMD terms had been compensated for. Here, the equivalence between the previous scheme and the new orthogonal scheme is thus verified for third-order IMD.

Let us assume $I^{\prime}$ and $Q^{\prime}$ are the output of the IQ-modulator of the inter-band stage, and then

$$
\begin{aligned}
I^{\prime} & =\alpha I-\beta Q \\
& =\left\{\operatorname{Re}\left(\alpha_{3}\right) E^{2}-\operatorname{Im}\left(\alpha_{3}\right) \widehat{E^{2}}\right\} I-\left\{\operatorname{Re}\left(\beta_{3}\right) E^{2}-\operatorname{Im}\left(\beta_{3}\right) \widehat{E^{2}}\right\} Q
\end{aligned}
$$

$$
\begin{aligned}
Q^{\prime} & =\beta I+\alpha Q \\
& =\left\{\operatorname{Re}\left(\beta_{3}\right) E^{2}-\operatorname{Im}\left(\beta_{3}\right) \widehat{E^{2}}\right\} I-\left\{\operatorname{Re}\left(\alpha_{3}\right) E^{2}-\operatorname{Im}\left(\alpha_{3}\right) \widehat{E^{2}}\right\} Q .
\end{aligned}
$$

In the general case, we have

$$
\begin{aligned}
& \alpha=\frac{Z_{1}-j Z_{2}}{2} \\
& \beta=\frac{Z_{2}+j Z_{1}}{2} .
\end{aligned}
$$

For the lower sideband comparison, let us assume that $\alpha_{3}=$ $Z_{1}$ and $\beta_{3}=j Z_{1}$, and then we have

$$
\begin{aligned}
& \alpha_{3}=\operatorname{Re}\left(Z_{1}\right)+j \operatorname{Im}\left(Z_{1}\right) \\
& \beta_{3}=-\operatorname{Im}\left(Z_{1}\right)+j \operatorname{Re}\left(Z_{1}\right) .
\end{aligned}
$$

Therefore, we have

$$
\operatorname{Re}\left(\alpha_{3}\right)=\operatorname{Im}\left(\beta_{3}\right) ; \operatorname{Im}\left(\alpha_{3}\right)=-\operatorname{Re}\left(\beta_{3}\right) .
$$

Inserting (16) into (14) and (15) gives

$$
\begin{aligned}
I^{\prime}= & \operatorname{Re}\left(\alpha_{3}\right) E^{2} I-\operatorname{Im}\left(\alpha_{3}\right) \widehat{E^{2}} I \\
& -\operatorname{Re}\left(\beta_{3}\right) E^{2} Q+\operatorname{Re}\left(\beta_{3}\right) \widehat{E^{2}} Q \\
= & \operatorname{Re}\left(\alpha_{3}\right) E^{2} I-\operatorname{Im}\left(\alpha_{3}\right) \widehat{E^{2}} I \\
& -\operatorname{Im}\left(\alpha_{3}\right) E^{2} Q+\operatorname{Re}\left(\alpha_{3}\right) \widehat{E^{2}} Q \\
Q^{\prime}= & \operatorname{Re}\left(\alpha_{3}\right) E^{2} Q-\operatorname{Im}\left(\alpha_{3}\right) \widehat{E^{2}} Q \\
& +\operatorname{Re}\left(\beta_{3}\right) E^{2} I-\operatorname{Im}\left(\beta_{3}\right) \widehat{E^{2}} I \\
= & \operatorname{Re}\left(\alpha_{3}\right) E^{2} Q-\operatorname{Im}\left(\alpha_{3}\right) \widehat{E^{2}} Q \\
& -\operatorname{Im}\left(\alpha_{3}\right) E^{2} I-\operatorname{Re}\left(\alpha_{3}\right) \widehat{E^{2}} I .
\end{aligned}
$$

After a few algebra steps, we obtain

$$
\begin{aligned}
I^{\prime}= & \operatorname{Re}\left(\alpha_{3}\right)\left(E^{2} I+\widehat{E^{2}} Q\right)-\operatorname{Im}\left(\alpha_{3}\right)\left(\widehat{E^{2}} I-E^{2} Q\right) \\
Q^{\prime}= & -\operatorname{Im}\left(\alpha_{3}\right)\left(E^{2} I+\widehat{E^{2}} Q\right)-\operatorname{Re}\left(\alpha_{3}\right)\left(\widehat{E^{2}} I-E^{2} Q\right) \\
& \times E^{2} I+\widehat{E^{2}} Q \\
= & 2\left(I_{U} I_{L}-\hat{I_{U}} \hat{I_{L}}\right)\left(I_{U}+I_{L}\right) \\
& +2\left(I_{U} \hat{I_{L}}+\hat{I_{U}} I_{L}\right)\left(Q_{U}+Q_{L}\right) \\
= & 2\left(I_{U}^{2} I_{L}-I_{U} \hat{I_{L}} I_{L}+I_{U} I_{L}^{2}-\hat{I}_{L} \hat{I_{U}} \hat{I_{L}}\right) \\
& +2\left(I_{U} \hat{I_{U}} \hat{I}_{L}+\hat{I}_{L}{\hat{I_{U}}}^{2}-{I_{U}}^{2}-I_{L} \hat{I_{U}} \hat{I_{L}}\right) \\
= & 2\left(I_{U}^{2}+{\hat{I_{U}}}^{2}\right) I_{L}+2\left(\hat{I}_{L}^{2}-\hat{I}_{L}{ }^{2}\right) I_{U}-4 I_{L} \hat{I_{U}} \hat{I_{L}} \\
= & 2\left\{E_{U}^{2} I_{L}+\left(I_{L}^{2}-Q_{L}^{2}\right) I_{U}+2 I_{L} Q_{U} Q_{L}\right\} \\
= & 2\left\{E_{U}^{2} I_{L}+I_{L}(3)\right\} .
\end{aligned}
$$


Similarly, we obtain

$$
\begin{aligned}
\widehat{E^{2} I-} & E^{2} Q \\
= & 2\left(I_{U} \hat{I_{L}}+\hat{I_{U}} I_{L}\right)\left(I_{U}+I_{L}\right) \\
& -2\left(I_{U} I_{L}-\hat{I_{U}} \hat{I_{L}}\right)\left(Q_{U}+Q_{L}\right) \\
= & 2\left(-I_{U} Q_{L}+Q_{U} I_{L}\right)\left(I_{U}+I_{L}\right) \\
& -\left(I_{U} I_{L}+Q_{U} Q_{L}\right)\left(Q_{U}+Q_{L}\right) \\
= & 2\left\{-I_{U}^{2} Q_{L}+Q_{U} I_{U} I_{L}-I_{U} I_{L} Q_{L}+Q_{U} I_{L}^{2}\right. \\
& \left.-I_{U} I_{L} Q_{U}-Q_{U}^{2} Q_{L}-I_{U} I_{L} Q_{L}-Q_{U} Q_{L}^{2}\right\} \\
= & -2\left\{E_{U} Q_{L}+2 I_{U} I_{L} Q_{L}-\left(I_{L}^{2}-Q_{L}^{2}\right) Q_{U}\right\} \\
= & -2\left\{E_{U}^{2} Q_{L}+Q_{L}(3)\right\} .
\end{aligned}
$$

For the upper sideband, let us assume $\alpha_{3}=Z_{2}$ and $\beta_{3}=$ $-j Z_{2}$, and then we have

$$
\begin{gathered}
\alpha_{3}=\operatorname{Re}\left(Z_{2}\right)+j \operatorname{Im}\left(Z_{2}\right), \\
\beta_{3}=\operatorname{Im}\left(Z_{2}\right)-j \operatorname{Re}\left(Z_{2}\right) .
\end{gathered}
$$

After same simple algebra, we obtain

$$
\begin{aligned}
I^{\prime} & =\operatorname{Re}\left(\alpha_{3}\right)\left(E^{2} I-\widehat{E^{2}} Q\right)-\operatorname{Im}\left(\alpha_{3}\right)\left(\widehat{E^{2}} I+E^{2} Q\right) \\
Q^{\prime} & =\operatorname{Im}\left(\alpha_{3}\right)\left(E^{2} I-\widehat{E^{2}} Q\right)+\operatorname{Re}\left(\alpha_{3}\right)\left(\widehat{E^{2}} I+E^{2} Q\right) .
\end{aligned}
$$

The terms $E^{2} I-\widehat{E^{2}} Q$ and $\widehat{E^{2}} I+E^{2} Q$ are given by the following equations:

$$
\begin{aligned}
& E^{2} I-\widehat{E^{2}} Q=2\left\{E_{L}^{2} I_{U}+I_{U}(3)\right\} \\
& \widehat{E^{2}} I+E^{2} Q=2\left\{E_{L}^{2} Q_{U}-Q_{U}(3)\right\} .
\end{aligned}
$$

The terms $E_{U}^{2} I_{L}, E_{U}^{2} Q_{L}, E_{L}^{2} I_{U}$, and $E_{L}^{2} Q_{U}$, appearing in the interband terms given by (16)-(19), respectively, are undesirable in-band terms arising in the original nonorthogonal scheme [3] which are now automatically eliminated by the new orthogonal scheme.

\section{APPENDIX II \\ NONLINEAR IMD DEFINITION}

It is interesting to consider the impact of a fifth-order expansion on the in-band third-order IMD. For this purpose, we assume that the lower (L) and upper (U) bands consist each of two tones $\mathrm{a}$ and $\mathrm{b}$ as

$$
x_{L}=x_{L a}+x_{L b} \quad x_{U}=x_{U a}+x_{U b}
$$

where we use the compact notation

$$
x_{p}=I_{p}+j Q_{p}
$$

The tone frequencies are also selected to have the same frequency separation: $f_{L b}-f_{L a}=f_{U b}-f_{U a}$. Further, we shall assume that the phase of these various signals $x_{L a}, x_{L b}, x_{U a}$ and $x_{U b}$ are statistically uncorrelated to remove any sweet point.
The third-order lower intermodulation $\mathrm{IMD}_{3, L}$ at the frequencies $f$ is then given by the ratio of the following expected values after the average over the signal phases is performed:

$$
\begin{aligned}
& \mathrm{IMD}_{3, L}\left(2 f_{L b}-f_{L a}\right)=\mathrm{IMD}_{3, L}\left(2 f_{L a}-f_{L b}\right) \\
& =\left\{\left|\frac{\partial \gamma_{-1}\left(\left\langle E_{U}^{2}\right\rangle,\left\langle E_{L}^{2}\right\rangle\right)}{\partial E_{L}^{2}}\right|^{2}\left\langle\left|x_{L a}^{*} x_{L b}^{2}\right|^{2}\right\rangle\right. \\
& \left.+\left|\frac{\partial \gamma_{-1}\left(\left\langle E_{U}^{2}\right\rangle,\left\langle E_{L}^{2}\right\rangle\right)}{\partial E_{U}^{2}}\right|^{2}\left\langle\left|x_{L b} x_{U a}^{*} x_{U b}\right|^{2}\right\rangle\right\} \\
& \times\left\{\left|\gamma_{-1}\left(\left\langle E_{U}^{2}\right\rangle,\left\langle E_{L}^{2}\right\rangle\right)\right|^{2}\left\langle\left|x_{L b}\right|^{2}\right\rangle\right\}^{-1} \\
& \mathrm{IMD}_{3, U}\left(2 f_{U b}-f_{U a}\right)=\operatorname{IMD}_{3, U}\left(2 f_{U a}-f_{U b}\right) \\
& =\left\{\left|\frac{\partial \gamma_{1}\left(\left\langle E_{U}^{2}\right\rangle,\left\langle E_{L}^{2}\right\rangle\right)}{\partial E_{L}^{2}}\right|^{2}\left\langle\left|x_{U b} x_{L a}^{*} x_{L b}\right|^{2}\right\rangle\right. \\
& \left.+\left|\frac{\partial \gamma_{1}\left(\left\langle E_{U}^{2}\right\rangle,\left\langle E_{L}^{2}\right\rangle\right)}{\partial E_{U}^{2}}\right|^{2}\left\langle\left|x_{U a}^{*} x_{U b}^{2}\right|^{2}\right\rangle\right\} \\
& \times\left\{\left|\gamma_{1}\left(\left\langle E_{U}^{2}\right\rangle,\left\langle E_{L}^{2}\right\rangle\right)\right|^{2}\left\langle\left|x_{U b}\right|^{2}\right\rangle\right\}^{-1}
\end{aligned}
$$

where we define

$$
\gamma_{p}=\alpha_{p}\left(E_{U}^{2}, E_{L}^{2}\right)+j \beta_{p}\left(E_{U}^{2}, E_{L}^{2}\right)
$$

and use the notation $E_{p}=\left|x_{p}\right|$ so that, for example, $E_{L b}=$ $\left|x_{L b}\right|$. In the previous expansion, use was made of the average envelope

$$
\left\langle E_{L}^{2}\right\rangle=E_{L a}^{2}+E_{L b}^{2} \quad\left\langle E_{U}^{2}\right\rangle=E_{U a}^{2}+E_{U b}^{2}
$$

assuming the signals $x_{L a}, x_{L b}, x_{U a}$, and $x_{U b}$ to be statistically uncorrelated, as in the case in multiband and multicarrier communication systems.

At low input power levels, $\left\langle\left. E_{L}\right|^{2}\right\rangle$ and $\left\langle E_{U}^{2}\right\rangle$ are small, and the third-order IMDs in (20) reduce to

$$
\begin{aligned}
\operatorname{IMD}_{3, L}\left(2 f_{L b}-f_{L a}\right) & =\operatorname{IMD}_{3, L}\left(2 f_{L a}-f_{L b}\right) \\
& \simeq \frac{\left|\gamma_{-1,1,0}\right|^{2}}{\left|\gamma_{-1,0,0}\right|^{2}} E_{L a}^{2} E_{L b}^{2}+\frac{\left|\gamma_{-1,0,1}\right|^{2}}{\left|\gamma_{-1,0,0}\right|^{2}} E_{U a}^{2} E_{U b}^{2} \\
\operatorname{IMD}_{3, U}\left(2 f_{U b}-f_{U a}\right) & =\operatorname{IMD}_{3, U}\left(2 f_{U a}-f_{U b}\right) \\
& \simeq \frac{\left|\gamma_{1,1,0}\right|^{2}}{\left|\gamma_{1,0,0}\right|^{2}} E_{L a}^{2} E_{L b}^{2}+\frac{\left|\gamma_{1,0,1}\right|^{2}}{\left|\gamma_{1,0,0}\right|^{2}} E_{U a}^{2} E_{U b}^{2}
\end{aligned}
$$

Clearly, at low input average power, the third-order IMDs for the lower and upper bands of (21) and (22) are not affected by the fifth-order nonlinearites. However the 5th order nonlinearities will contribute to the power amplifier output at high enough average input power via the dependence of the $\gamma_{p}$ coefficients upon $\left\langle E_{L}^{2}\right\rangle$ and $\left\langle E_{L}^{2}\right\rangle$. For a 5 th order system the threshold at which the 5th order nonlinearities must be accounted will depends on the magnitude of the coefficients $\gamma_{ \pm 1,2,0}, \gamma_{ \pm 1,0,2}$, and 
$\gamma_{ \pm 1,1,1}$ as well as the amplitude of $\left\langle\left. E_{L}\right|^{2}\right\rangle$ and $\left\langle E_{U}^{2}\right\rangle$. Assuming $E_{L a}^{2}=E_{L b}^{2}=E_{U a}^{2}=E_{U b}^{2}=\left\langle E_{L / U}^{2}\right\rangle / 2$ an upper bound on the power level at which the 5th order nonlinearities would be comparable to the 3 rd order nonlinearities is given by:

$$
\left\langle E_{L / U}^{2}\right\rangle=\frac{\min \left\{\gamma_{ \pm 1,1,0}, \gamma_{ \pm 1,0,1}\right\}}{\max \left\{\gamma_{ \pm 1,2,0}, \gamma_{ \pm 1,0,2}, \gamma_{ \pm 1,1,1}\right\}}
$$

The presence of a noticeable fifth-order intermodulation at the interband frequencies $3 f_{U a / b}-2 f_{L a / b}$ and $3 f_{L a / b}-2 f_{U a / b}$ as is shown in Fig. 10(b) provides a measure of the contribution of the fifth-order in-band nonlinearities at the in-band frequencies $2 f_{L b}-f_{L a}, 2 f_{L a}-f_{L b}, 2 f_{U b}-f_{U a}$ and $2 f_{U a}-f_{U b}$. In Fig. 10(b), accounting for the fifth-order nonlinearities enables ones to reduce the ACPR by a factor $5 \mathrm{~dB}$, as was verified in the tuning process. In Section II-C, a fifth-order linearization was demonstrated to provide up to $10 \mathrm{~dB}$ of improvement in ACPR reduction over a third-order linearization.

\section{ACKNOWLEDGMENT}

The authors would like to thank the Altera Corporation for the donation of the DE3 FPGA testbed which was used in this work. The authors would also like to thank the reviewer for the suggestions that greatly improved the presentation of this paper. The authors would also like to thank Dr. C. Quindroit for many discussions.

\section{REFERENCES}

[1] J. Kim and K. Konstantinou, "Digital predistortion of wideband signals based on power amplifier model with memory," IEEE Electron. Lett., vol. 37 , no. 23, pp. 1417-1418, Nov. 2001.

[2] L. Ding, G. T. Zhou, D. R. Morgan, Z. Ma, J. S. Kenney, J. Kim, and C. R. Giardina, "A robust digital basedband predistorter constructed using memory polynomials," IEEE Trans. Commun., vol. 52, no. 1, pp. 159-165, Jan. 2004

[3] P. Roblin, S. K. Myoung, D. Chaillot, Y. G. Kim, A. Fathimulla, J. Stahler, and S. Bibyk, "Frequency-selective predistortion linearization of RF power amplifiers," IEEE Trans. Microw. Theory Tech., vol. 56, no. 1, pp. 65-76, Jan. 2008.

[4] X. Yang, P. Roblin, D. Chaillot, S. Mutha, J. Strahler, J. Kim, M. Ismail, J. Wood, and J. Volakis, "Fully orthogonal multi-carrier predistortion linearization for RF power amplifiers," in IEEE MTT-S Int. Microw. Symp. Dig., Boston, MA, Jun. 2009, pp. 1077-1080.

[5] P. Roblin, Nonlinear RF Circuits and Nonlinear Vector Network Analyzers: Interactive Measurement and Design Techniques. Cambridge, U.K.: Cambridge Univ., 2011.

[6] J. Kim, P. Roblin, X. Yang, and D. Chaillot, “A new architecture for frequency-selective digital predistortion linearization for RF power amplifiers," in IEEE MTT-S Int. Microw. Symp. Dig., Montreal, QC, Canada, Jul. 2012, pp. 1-3.

[7] A. Cidronali, I. Magrini, R. Fagotti, and G. Manes, "A new approach for concurrent dual-band if digital predistortion: System design and analysis," in Proc. Integr. Nonlinear Microw. Millimeter-Wave Circuits Workshop, Nov. 2008, pp. 127-130.

[8] S. A. Bassam, M. Helaoui, and F. M. Ghannouchi, "2-D digital predistortion (2-D-DPD) architecture for concurrent dual-band transmitters," IEEE Trans. Microw. Theory Tech., vol. 59, no. 10, pp. 2547-2553, Oct. 2011.

[9] S. A. Bassam, W. Chen, M. Helaoui, F. M. Ghannouchi, and Z. Feng, "Linearization of concurrent dual-band power amplifier based on 2D-DPD technique," IEEE Microw. Wireless Compon. Lett., vol. 21, no. 12, pp. 685-687, Dec. 2011.

[10] R. N. Braithwaite, "Digital predistortion of a power amplifier for signals comprising widely spaced carriers," in Proc. 78th Microw. Meas. Symp. , 2011, pp. 1-4.
[11] "16-Bit, 1.0 GSPS 2x-4x Interpolating Dual-Channel Digital-To-Analog Converter (DAC),” Texas Inst., 2008.

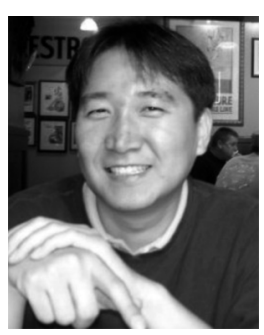

Jiwoo Kim (S'10) was born in Kyunggi, South Korea, in April 1975. He received the B.S. degree in electronics engineering from Sungkyunkwan University, Suwon, Korea, in 1998, the M.S. degree in electronics and electrical engineering from Pohang University of Science and Technology (POSTECH), Pohang, Korea, in 2000, and the Ph.D. degree in electrical and computer engineering from The Ohio State University, Columbus, in 2012.

From 2000 to 2006, he was with Solid Technologies, Inc., Seoul, Korea, where he developed RF and DSP algorithms and implementations for various digital repeater systems. In 2011, he was an Intern with Broadcom Corporation, Irvine, CA. He is currently with Apple, Inc., Cupertino, CA. His research interests include design and implementation of digital communication systems with emphasis on RF engineering.

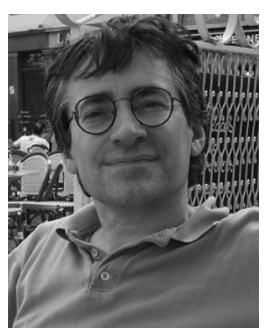

Patrick Roblin (M'85) was born in Paris, France, in September 1958. He received the Maitrise de Physics degree from the Louis Pasteur University, Strasbourg, France, in 1980, and the M.S. and D.Sc. degrees in electrical engineering from Washington University, St. Louis, MO, in 1982 and 1984, respectively.

In 1984, he joined the Department of Electrical Engineering, The Ohio State University (OSU), Columbus, as an Assistant Professor and is currently a Professor. His present research interests include the measurement, modeling, design, and linearization of nonlinear RF devices and circuits such as oscillators, mixers, and power amplifiers. He is the first author of two textbooks, High-Speed Heterostructure and Devices (Cambridge University Press, 2002) and Nonlinear RF Circuits and Nonlinear Vector Network Analyzers (Cambridge University Press, 2011). At OSU, he is the founder of the Non-Linear RF Research Lab. At OSU, he has developed two educational $\mathrm{RF} / \mathrm{microwave}$ laboratories and associated curriculum for training both undergraduate and graduate students.

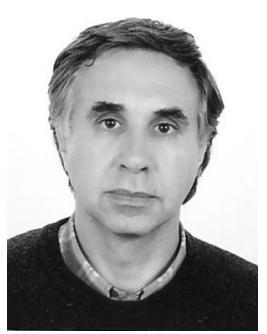

Dominique Chaillot was born in Brive, France, in October 1963. He received the Maitrise de Physique degree from the Université des sciences, Laboratoire IRCOM, Limoges, France, in 1985, the Ph.D. degree in electrical engineering from IRCOM (now XLIM) Limoges, France, in 1989, and the M.B.A. degree from IAE Sorbonne, Paris, France, in 1992.

In 1990, he joined the Commissariat à l'Énergie atomique (French Atomic Agency), Bruyère le Chatel, France, as a Research Engineer, and then he joined the CESTA, Le Barp, France, in 1997. In 2003 and 2008, he was with the Department of Electrical and Computer Engineering, The Ohio State University, Columbus, as an Invited Scholar. His expertise is on real-time signal processing in RF systems. His current research interests include the measurement design and linearization of nonlinear RF devices and power amplifiers.

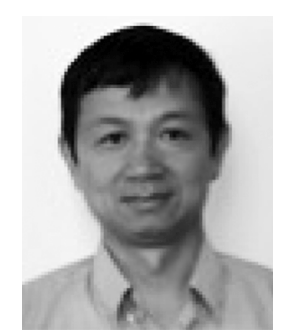

Zhijian Xie (M'01) received the B.S and M.S. degrees in solid-state physics from the University of Science and Technology of China, Hefei, China, in 1992 and 1995, respectively, and the Ph.D. degree in electrical engineering from Princeton University, Princeton, NJ, in 2001.

After working with Agere systems, RF Micro Devices, and DSM Solutions, he joined North Carolina Agricultural \& Technical State University, Greensboro, in 2009. He holds three U.S. patents and has one pending. His current research interests include microwave/RF integrated circuits and devices, electrostatic discharge protection, novel devices, and biomedical devices and modeling. 